\title{
On the Symbolic Manipulation and Code Generation for Elasto-Plastic Material Matrices
}

T.Y. Chang and A.F. Saleeb

University of Akron

Akron, Ohio

and

P.S. Wang and H.Q. Tan

Kent State University

Kent, Ohio

October 1991

Prepared for

Lewis Research Center

Under Grants NAG3-307 and NAG3-298

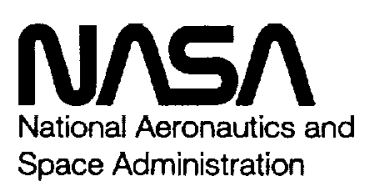

(NASA-CR-197223) ON THE SYMBOLIC

N92-11375

MANIPULATION AND CODE GENERATION FOR

ELASTO-PLASTIC MATERIAL MATRICES FInal

Report (akron Univ.) $29 \mathrm{p}$

CSCL $20 \mathrm{~K}$ 


\section{INTRODUCTION}

Development of new constitutive models for finite element applications represents a very important area of research in engineering disciplines. This is evidenced by research activities, for example, associated with high temperature composites [1,2], reinforced concrete [3], geotechnical materials $[4,5]$. The efforts in constitutive research involve the development of mathematical relationships for predicting nonlinear response of materials, derivation of material stiffness matrix appropriate for finite element calculations, computer implementation, and finally, coding verifications. Obvious$1 y$, the entire process requires a great deal of manual algebraic manipulations and computer programing. Hence, the response time for the related efforts is quite long, in the order of many months. As a result, it is rather difficult for the researcher to introduce any significant changes or modifications into the constitutive theory, since the required effort is rather tedious. Moreover, the outcome of research work" may be affected by human errors which are often difficult to detect. In view of this discussion, it is apparent that symbolic manipulations can provide a significant incentive to the development of constitutive theories and their finite element applications. With the availability of MACSYMA or VAXIMA (i.e. VAX computer version of MACSYMA), it becomes possible to derive the required material matrix of a constitutive model in an efficient way. The obvious advantages of using VAXIMA are several: i) reduce manual tedium for deriving the material stiffness matrix, ii) improved reliability of analysis results, iii) quick response time for constitutive model development. However, direct application of VAXIMA will not be trouble-free. One major obstacle is the exponential growth of algebraic expressions during intermediate derivations, which requires significant storage space and increased computer time. Moreover, it is also 
possible to convert the derived expressions directly into Fortran coding. Then problems associated with modularity and interface with the main program must be addressed.

Application of symbolic manipulations to finite element analys is is not new. Most of the previous work was concentrated, however, on the derivation of element stiffness and mass matrices [6-11]. No published work was found on the application of this procedure to the development of material matrices, although the general concept is somewhat similar. The objective of our research is to use symbolic manipulations for the derivation of a class of material matrices for finite element analysis; namely, elasto-plastic materials. The scope of our work includes derivation of material matrices and automatic Fortran code generation. In this paper, we will demonstrate a systematic application of the symbolic mathematical package, VAXIMA, the method of expression simplifications, and code generation in the form of RATFOR. Three sample constitutive models are included to illustrate the procedures developed. They are: von Mises metal plasticity, Drucker-Prager soil plasticity model, and a plasticity-based model for concrete. These models have been extensively used for different finite element analyses in structural and geotechnical engineering fields. 


\section{THEORETICAL EQUATIONS}

For the sake of discussion, the stress-strain equations for elastoplastic materials are briefly outlined in this section. More detailed descriptions of these relations can be found in several texts [e.g., 12-14]. It is noted that our primary purpose here is to derive the general form of the material stiffness matrix as commonly used in the displacement-based finite element analysis.

The first basic assumptions in the incremental (flow) theory of plasticity is the additive decomposition of the total incremental strain vector, $d \varepsilon$, into elastic and $p l a s t i c$ components, $\underset{\sim}{d \varepsilon^{e}}$ and $\underset{\sim}{d \varepsilon^{p}}$, respectively. In addition, the incremental elastic strain components are often assumed to be linearly related to the increment of stress vector (generalized form of Hooke's Law),

$$
\underset{\sim}{d \sigma}=\underset{\sim}{C} \underset{\sim}{E}(\underset{\sim}{d}-\underset{\sim}{p})
$$

where $\underset{\sim}{C E}$ is an elastic material stiffness matrix.

Thus, the main task in the formulation of the elasto-plastic model is concerned with establishing the manner in which the plastic strain increments are related to the stress increment vector and the history of deformation. To this end, three fundamental assumptions of plasticity theory are employed. These are: i) the yield (loading) function defining the limit of elasticity of the material during the course of plastic deformations ii) an appropriate hardening rule specifying the manner for the evolution of the yield surfaces during plastic straining (e.g. isotropic, kinematic, or mixed hardening, etc) and $i i i)$ a flow rule that provides the general form of the incremental plastic stress-strain relationships (e.g. associated flow or normality rule, or the non-associated flow rule). 
Under isothermal conditions, the yield function is expressed as

$$
f=f(\sigma, k)
$$

where $\sigma$ is the stress vector, and $k$ represents a strain-hardening parameter which may vary as a function of plastic deformation history or other state variables. Note that, in general, one or more strain-hardening parameters may be used, and these may actually be scalars (e.g., accumulated plastic work) or tensorial quantities (e.g., plastic strain components). However, for convenience, we only use one scalar hardening parameter here, since all of the specific plasticity models considered are of this type. Also, associated flow rule is employed in the three models discussed.

Adopting the normality rule, the plastic flow (or increment of plastic strain vector) is given by

$$
\underset{\sim}{\mathrm{d} \varepsilon^{\mathrm{p}}}=\mathrm{d \lambda} \frac{\partial \mathrm{f}}{\partial \underset{\sim}{\sigma}}
$$

where $d \lambda$ is a positive scalar quantity often referred to as the loading parameter or plastic multiplier, which generally depends on the current state of stress $\underset{\sim}{\sigma}$, incremental stresses $\underset{\sim}{\mathrm{d}}$, and loading history.

Based on the above relationships, and employing the so-called consistency condition [13], one can easily derive the general form of the incremental stress-strain equations for a material undergoing plastic deformations $[12,14]$; that is

$$
\underset{\sim}{d \sigma}=\underset{\sim}{E P} d \varepsilon
$$

where $\underset{\sim}{c^{E P}}$ designates the familiar elasto-plastic material stiffness matrix which has the form

$$
\underset{\sim}{c} \underset{\sim}{E P}=\underset{\sim}{c}-\underset{\sim}{c}
$$

where $C^{P}$ is a plastic matrix given by 


$$
\underset{\sim}{c^{p}}=\frac{\stackrel{\sim}{\sim}^{E} \cdot\left(\frac{\partial f}{\partial g}\right)\left(\stackrel{\sim}{C}^{E} \cdot \frac{\partial f}{\partial g}\right)^{\top}}{\left(\frac{\partial f}{\partial \sigma}\right)^{\top}{\underset{\sim}{C}}^{E}\left(\frac{\partial f}{\partial \sigma}\right)+\left(\frac{\partial f}{\partial \sigma}\right)^{\top}\left(\frac{\partial f}{\partial \underset{\sim}{\epsilon}} p\right)}
$$

In addition to the relationship in Eq. (4), the matrix $\underset{\sim}{C}$ is also used for the evaluation of element stiffness matrix $\underset{\sim}{k}=\iiint \underset{\sim}{B} \underset{\sim}{C} \underset{\sim}{B} d v$.

It is seen from Eq. (6) that in order to obtain specific expression for the elasto-plastic matrix $C^{E P}$, one has to manipulate the derivatives of yield function with respect to $\underset{\sim}{\sigma}$ and $\underset{\sim}{\varepsilon}$ and then carry out appropriate matrix multiplications. For a complex mathematical expression of $f$, the associated manipulation can be quite tedius if it is done manually. In the next section, we outline the procedure through which this can be done conveniently by symbolic manipulations using VAXIMA. 


\section{SYMBOLIC MANIPULATIONS}

In order to find the explicit expression of elasto-plastic matrix, i.e., Eq. (5), for a given material model, four types of mathematical manipulations need to be employed; i) differentiations of the yield function with respect to the stress or other variables, ii) matrix multiplications, iii) grouping of like-terms, and iv) expression simplifications. It was pointed out earlier that in most cases the results obtained from direct application of VAXIMA would not be useful due to the problem associated with expression growth. For this reason, a strategy must be developed to obtain an optimal (or simp-

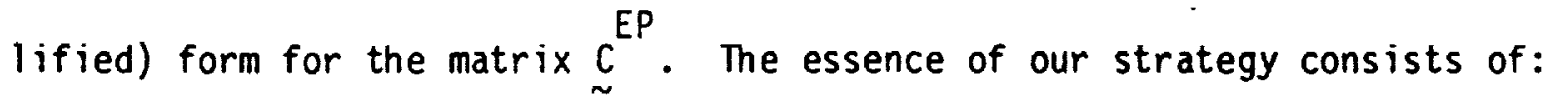

1. A structured derivation procedure to avoid redundant manipulations and to minimize expression growth.

2. Factorization and expression simplification through user intervention with interactive coding.

3. Introduction of intermediate variables.

4. Taking advantage of permutation and symmetry relationships of the terms and matrix involved during intermediate derivations.

With the above guidelines in mind, the derivation of elasto-plastic material matrix, i.e. Eq. (5), involves the following:

1. Finding the derivatives

$$
\mathrm{p}=\left(\frac{\partial f}{\partial \sigma}\right)=\left(\frac{\partial f}{\partial \sigma_{11}}, \frac{\partial f}{\partial \sigma_{22}},{\frac{\partial f}{\partial \sigma_{33}}}_{33} \frac{\partial f}{\partial \sigma_{12}},{\frac{\partial f}{\partial \sigma_{23}}}_{2}, \frac{\partial f}{\partial \sigma_{31}}\right)
$$

and

$$
\underset{\sim}{q}=(\underset{\sim \underset{\sim}{g} p}{\underbrace{}_{f}})=\left(\frac{\partial f}{\partial \varepsilon_{11}^{p}}, \frac{\partial f}{\partial \varepsilon_{22}^{p}}, \frac{\partial f}{\partial \varepsilon_{33}^{p}}, \frac{1}{2} \frac{\partial f}{\partial \varepsilon_{12}^{p}}, \frac{1}{2} \frac{\partial f}{\partial \varepsilon_{23}^{p}}, \frac{1}{2} \frac{\partial f}{\partial \varepsilon_{31}^{p}}\right)
$$


2. Performing matrix multiplications for the numerator of $\underset{\sim}{C^{P}}$ :

$$
\underset{\sim}{E} \cdot \underset{\sim}{P}
$$

and for the denominator:

$$
\underset{\sim}{p} \underset{\sim}{C^{E}} \stackrel{p}{\sim} \text { and } \underset{\sim}{p} \cdot \underset{\sim}{q}
$$

3. Conducting expression simplifications during the course of derivations. Two simplification conditons are often used for substitutions:

$$
S_{11}+S_{22}+S_{33}=0
$$

and

$$
s_{11}^{2}+s_{22}^{2}+s_{33}^{2}+2\left(s_{12}^{2}+s_{23}^{2}+s_{31}^{2}\right)=2 J_{2}
$$

In the sequel, three specific examples of plasticity material models are employed to demonstrate our procedure outlined in the above.

\section{1 von Mises Metal Plasticity Model}

We consider first the von Mises model with isotropic strain hardening for metal plasticity as an example to demonstrate our procedure. In this case, the yield function $f$ is given by

$$
f=\frac{1}{2} S^{\top} \underset{\sim}{S}-k^{2}
$$

where $S$ represents the vector of stress deviators and

$$
\dot{S}=\underset{\sim}{\sigma}-\sigma_{m} \cdot \delta
$$

$\delta$ is a vector of Kroneckle delta

$$
\delta=(1,1,1,0,0,0)
$$


and $\sigma_{m}$ is the mean stress, a scalar quantity given by

$$
\sigma_{m}=\frac{1}{3}\left(\sigma_{11}+\sigma_{22}+\sigma_{33}\right)
$$

The parameter $k$ is a function of plastic work

$$
k=k\left(w_{p}\right)
$$

Also note that $k$ is related to the effective stress $\sigma$ by [12]

$$
k=\frac{1}{3} \sigma_{e}
$$

Using VAXIMA, at first we evaluate

$$
\underset{\sim}{p}=\left(s_{11}, S_{22}, s_{33}, 2 s_{12}, 2 S_{23}, 2 S_{31}\right)
$$

and

$$
\underset{\sim}{q}=H\left(\sigma_{11}, \sigma_{22}, \sigma_{33}, \sigma_{12}, \sigma_{23}, \sigma_{31}\right)
$$

In the above, the common factor $H$ is the so-called plastic modulus and it is found to be

$$
H=-\frac{2}{3} \quad \sigma_{e} \frac{\partial f}{\partial w_{p}}
$$

Next, evaluating

$$
\begin{aligned}
\underset{\sim}{Y} & =\underset{\sim}{c} \cdot \underset{\sim}{p} \\
& =\frac{E}{(1+v)(1-2 v)}\left[\begin{array}{c}
(1-v) S_{11}+v S_{22}+v S_{33} \\
v S_{11}+(1-v) S_{22}+v S_{33} \\
v S_{11}+v S_{22}+(1-v) S_{33} \\
\left(1-2 v S_{12}\right. \\
\left(1-2 v S_{23}\right. \\
(1-2 v) S_{31}
\end{array}\right]
\end{aligned}
$$

The numerator of $\underset{\sim}{c^{p}}$ is equal to

$$
\underset{\sim}{\gamma} \cdot \underset{\sim}{\gamma}=4 G^{2} \cdot{\underset{\sim 2}{S}}^{\top}
$$


where $G$ is the shear modulus, and $S_{2}$ is a $6 \times 6$ matrix in wich the entries are the products of stress deviators, i.e.,

$$
\mathrm{S}_{2}=\underset{\sim}{S} \cdot \underset{\sim}{\mathrm{S}}
$$

Then, we evaluate the denominator of $c^{p}$. For this purpose, we utilize the two simplification conditions given in Eqs. (7) and (8). Thus, the following simplified expressions can be found

$$
\underset{\sim}{p} \underset{\sim}{C} \underset{\sim}{p}=\frac{4}{3} G \cdot \sigma_{e}^{2}
$$

and

$$
\underset{\sim}{p} \cdot \underset{\sim}{q}=\frac{2}{3} H \underset{e}{2}
$$

By combining Eqs (19), (21) and (22) with Eq. (5), we finally obtain

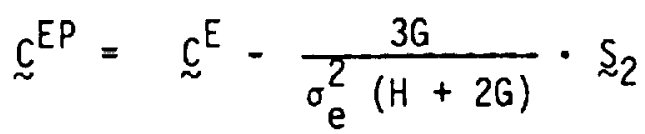

The above expression corresponds to that given in [5].

\subsection{Drucker-Prager Soil Plasticity Model}

We now consider a more complex material model; namely, the DruckerPrager, perfect-plasticity model used extensively for geotechnical materials. In addition to the above procedures, intermediate variables have to be introduced in this case in order to avoid the problem of expression growth. The yield function of Drucker-Prager model assumes the form [16]

$$
f=J_{2}^{1 / 2}+\alpha I_{1}-k
$$

where $I_{1}$ is the first stress invarient; $J_{2}$ is the second invarient of stress deviators; $\alpha$ and $k$ are material constants. 
If we follow the same procedure for the von Mises model without introducing any intermediate variables, growth of algebraic expressions becomes apparent. For example, the first three entries in the first row of $\underset{\sim}{\mathrm{P}}$ are listed in Fig. 1. There are twenty-one similar entries when the symmetry condition of $\underset{\sim}{P}$ is taken into consideration. However, after we have introduced the intermediate variables $\underset{\sim}{\mathrm{a}}$ and $\mathrm{ff}$,

$$
\begin{aligned}
a & =\left(a_{1}, a_{2}, \ldots a_{6}\right) \\
\sim & =\left(f f_{1}, f f_{2}, \ldots f f_{6}\right)
\end{aligned}
$$

where $a_{i}, f_{i}$ are defined in $F_{i g} .2$, then

$$
{\underset{\sim}{C P}}^{E P} \underset{\sim}{f f} \cdot \underset{\sim}{(f f)^{\top}}
$$

of course, in computer coding we only need to perform matrix multiplication for either the upper or lower half of $\underset{\sim}{c}$ EP owing to its symmetry properties.

\subsection{Concrete Plasticity Model}

The final illustrative example to be considered here is a concrete plasticity model proposed recently by Chen and Chen $[13,17]$. The derivation of elasto-plastic matrix for this hardening plasticity model is quite tedius due to its complex mathematical expressions. In fact, through the use of symbolic manipulations an error was found in the coefficient of the plastic matrix published in the literature in $[12,13,17]$. The error is associated with the shear stress terms, which usually do not appear for simple test cases like simple compression or biaxial compression tests that have been used in various model verifications. Moreover, the error terms do not appear when the model is reduced to the von Mises theory.

The yield function of the concrete plasticity model is given by 


$$
f=\frac{J_{2}+\frac{B}{3} I_{1}+n I_{1}^{2}}{1-\frac{\alpha}{3} I_{1}}-\tau^{2}=0
$$

where $\alpha$ and $B$ are material constants; $\tau$ is an effective stress; $n$, a parameter, whose definition varies with the stress state: $n=0$ for compression compression stress states; $n=-1 / 6$ for tension - compression or tension tension stress states.

For this model, the problem of expression growth becomes prohibitive if intermediate variables are not introduced. By successive manipulations with VAXIMA the following variables are identified:

$$
\begin{aligned}
& \underset{\sim}{a}=\frac{1}{m}(\rho \cdot \underset{\sim}{\delta}+\hat{S}) \\
& \underset{\sim}{S}=\left(S_{11}, S_{22}, S_{33}, 2 S_{12}, 2 S_{23}, 2 S_{31}\right) \\
& \underset{\sim}{\delta}=(1,1,1,0,0,0) \\
& \frac{1}{w}=\frac{1}{E^{2}}(u+v) \\
& u=\frac{H}{m}\left[2 J_{2}+3 \rho^{2}+2\left(S_{12}^{2}+S_{23}^{2}+S_{31}^{2}\right)\right]^{1 / 2} \\
& v=\frac{E_{c}}{m^{2}}\left[2 J_{2}+3 \rho^{2}+v\left(4 J_{2}+3 \rho^{2}\right)\right] \\
& E_{c}=\frac{E^{2}}{(1+v)(1-2 v)}
\end{aligned}
$$




$$
\begin{aligned}
& p=n I_{1}+\frac{1}{3}\left(\beta+\alpha \tau^{2}\right) \\
& m=1-\frac{\alpha}{3} I_{1}
\end{aligned}
$$

where $E=$ Young's modulus, $\nu=$ Poisson's ratio, and $H=$ plast ic work hardening paratmeter. It is noted in the above that the underlined terms for $u$ were missing in the published expression [12]. The addition of these terms was verified by both symbolic manipulations using VAXIMA and independent manual derivations.

Moreover, we introduce a vector $f f(i), i=1,2, \ldots, 6$, of which the first three components are given by

$$
\underset{\sim \sim}{f f}(i)=(\underset{\sim}{I}+\nu \underset{\sim}{\Omega}) \underset{\sim}{a}(i), \quad i=1,2 \text {, and } 3
$$

and the last three components are

$$
\underset{\sim}{f f}(i)=\left(\frac{1-2 v}{2}\right) \cdot \underset{\sim}{a}(i), \quad i=4,5 \text { and } 6 \text {. }
$$

where $I$ is a $3 \times 3$ identity matrix and

$$
\Omega=\left[\begin{array}{rrr}
-1 & 1 & 1 \\
1 & -1 & 1 \\
1 & 1 & -1
\end{array}\right]
$$

The above procedure has been written in LISP program language with direct access to the internal data structure of VAXIMA. Hence, the package can be used for the symbolic manipulations of any elasto-plastic material matrix with two special features: i) no expression growth problem, and ii) high efficiency in terms of CPU time. 


\section{AUTOMATIC CODE GENERATION}

There is a definite advantage to convert the generated symbolic expressions into FORTRAN statements for finite element computations. By doing so, not only can the manual effort be avoided, but also it provides increased reliability on the constitutive relations. Instead of generating FORTRAN directly, we have utilized a generator called GENTRAN (symbolic to numerical code GENerator/TRANslator)[18] which has the ability to produce a RATFOR or C program in the form of a subroutine or part of a subroutine. Subsequently, the FORTRAN statements are generated from the RATFOR through a preprocessor.

Several systems are available in converting symbolic expressions to FORTRAN statements, such as MACTRAN [19], VAXTRAN [20] and REDUCE [21]. The MACTRAN Package converts MACSYMA equations and other expressions into FORTRAN code, and provides a text processor which allows the derived FORTRAN code segments to be interspersed with fixed code frómi program skeletons. Similar features are given in the REDUCE and VAXTRAN systems, except that VAXTRAN was written specifically for VAXIMA. All these packages represent a first step towards providing an interface between symbolic manipulations and numerical computations. However, they do not provide a convenient way to generate statements such as declarations, control-flow structures, 1/0 statements, functions, and subroutines. These statements, in general, are necessary for generating a complete and efficient FORTRAN program. For this reason, we have chosen to use a package called GENTRAN which was written in FRANZ LISP under the VAXIMA environment.

The immediate concern in generating a subprogram to interact with a finite element code is the interface problem. To minimize such problems, we have designed a template file shown in Fig. 3 which is somewhat universal for 
various finite element codes. For a specific plasticity material model (such as the von Mises or Drucker-Prager model), the material matrix subroutine is completed by including the generated statements in the template file as indicated in Fig. 3.

To demonstrate how the RATFOR code is generated by GENTRAN, we consider again the von Mises model. First, we define the $S_{2}$ matrix according to Eq.(18). Secondly, the elasto-plastic matrix $\underset{\sim}{C E P}$ is evaluated from Eq. (23). Let

$$
\begin{aligned}
S(i) & =\text { components of stress deviator, } i=1,2, \ldots, 6 . \\
\operatorname{CE}(i, j) & =\text { elastic material matrix } \\
\operatorname{CEP}(i, j) & =\text { elasto-plastic matrix } \\
\operatorname{CP}(i, j) & =\text { plastic material matrix } \\
\text { FACTOR } & =\frac{3 G}{\sigma_{e}^{2}(H+2 G)}
\end{aligned}
$$

Then the RATFOR code for the von Mises model is given as follows:

1. for $(i=1 ; \quad i<=6 ; \quad i=i+1)$

2. for $(j=i ; j<=6 ; j=j+1)$

3. $C P(i, j)=F A C T O R * S(i) * S(j)$

4. for $(i=1 ; \quad i<=6 ; \quad i=i+1)$

5. for $(j=i ; \quad j<=6 ; j=j+1)$

6. $\operatorname{CEP}(i, j)=C E(i, j)-C P(i, j)$

7. $\operatorname{CEP}(j, i)=\operatorname{CEP}(i, j)$

The translated FORTRAN code can be found in the Appendix.

The RATFOR code of the Drucker-Prager model is slightly different from that of the von Mises model due to the use of intermediate variables. In this case, let 


$$
\begin{aligned}
F F(i) & =\text { intermediate variables as defined in Eq. }(26), i=1,2, \ldots, 6 . \\
\text { FACTOR } & =1 /(G+\alpha B) \\
B & =\frac{2}{3} \alpha G\left(\frac{1+v}{1-2 v}\right) \\
v & =\text { Poisson's ratio }
\end{aligned}
$$

Then the RATFOR code is

$$
\begin{aligned}
& \text { 1. for }(i=1 ; \quad i<=6 ; \quad i=i+1) \\
& \text { 2. for }(j=i ; \quad j<=6 ; j=j+1) \\
& \text { 3. } \operatorname{CP}(i, j)=F A C T O R \star F F(i) \star F F(j) \\
& \text { 4. for }(i=1 ; \quad i<=6 ; \quad i=i+1) \\
& \text { 5. for }(j=i ; \quad j<=6 ; j=j+1) \\
& \text { 6. } \operatorname{CEP}(i, j)=\operatorname{CE}(i, j)-\operatorname{CP}(i, j) \\
& \text { 7. } \operatorname{CEP}(j, i)=\operatorname{CEP}(i, j)
\end{aligned}
$$

The translated FORTRAN code for the Drucker-Prager model is given in the Appendix.

Finally, with the introduction of the intermediate variables in Eqs. (29)-(37), which were obtained through the factorization of VAXIMA, the RATFOR coding of the concrete model becomes identical to that of the DruckerPrager model. The corresponding FORTRAN code is listed in the Appendix. 


\section{CONCLUSION}

A systematic procedure to perform symbolic manipulations using VAXIMA and FORTRAN code generation of elasto-plastic material matrices for finite element applications has been developed. The unique features of the proposed procedure are: i) the problem of expression growth was alleviated by introducing intermediate variables and step-wise expression simplifications, ii) the material matrix is automatically converted into FORTRAN coding, and iii) the use of a template file to ease the interface problem. This procedure can be applied not only to plasticity models with associated flow rules, but also to models with non-associated flow rules.

The potential benefits of the proposed procedure are two-fold: i) it can avoid manual tedium for constitutive model development, and ii) it provides increased reliability on the model for finite element applications. The same concept can be extended to other types of constitutive model development. For example, in the finite element analys is of viscoplastic constitutive models, the formation of Jacobian matrix for numerical integration requires lengthy algebraic manipulations of the rate stress-strain equations. Such manipulations can be easily performed by a well-designed VAXIMA procedure. Once the mathematical relations are derived, automatic code generation should become apparent.

\section{ACKNOWLEDGEMENT}

The work reported herein is supported by NASA Lewis Research Center, Cleveland, Ohio. The project monitor of our work is Dr. C. C. Chamis. 


\section{REFERENCES}

1. "Nonlinear Constitutive Relations for High Temperature Application-1984", Proceedings of a symposium held at NASA Lewis Research Center, Cleveland, Ohio, June $15-17,1984$.

2. C.S. Desai and R.H. Gallagher (ed), Constitutive Laws for Engineering Materials: Theories and Application, International Conference Proceedings, John Wiley and Sons, U.K., 1983.

3. ASCE-ACI Task Committee, "State-of-the-Art Report on Finite Element Analys is of Reinforced Concrete", ASCE Special Publications, New York, 1982.

4. R.K. Yong and H.Y. Ko (ed), Limit Equilibrium, Plasticity and Generalized Stress-Strain in Geotechnical Engineering, ASCE Proceedings, New York, 1981.

5. G.N. Pande and 0.C. Zienkiewicz (ed), Soil Mechanics - Transient and Cyclic Loads, John Wiley and Sons, 1982.

6. W. Luft, J.M. Roesset and J.J. Conners, "Automatic Generation of Finite El iement Matrices", J. Structural Div., ASCE, 97 (ST1), pp. 349-363, 1971.

7. R.H. Gunderson and A. Cetiner, "Element Stiffness Matrix Generator", J. Structural Div., ASCE, 97 (STi), pp. 363-375, 1971.

8. M.M. Cecchi and C. Lami, "Automatic Generation of Stiffness Matrices for Finite Element Analys is", Int. J. Numer. Meth. Engng. 11, pp. 396-400, 1977.

9. A.K. Noor and C.M. Andersen, "Computerized Symbolic Manipulation in Stuctural Mechanics-Progress and Potential", Computers \& Structures, Vol 10, pp. 95-118, 1979.

10. A.R. Korncoff and S.J. Fenves, "Symbolic Generation of Finite Element Stiffness Matrices", Computers \& Structures, Vol. 10, pp. 119-124, 1979.

11. P.S. Wang, T.Y.P. Chang and J.A. van Hulzen, "Code Generation and Optimization for Finite Element Analysis", G. Goos and J. Hartmanis, (ed), EUROSAM 84 Conference Proceedings, Springer-Verlag, LNCS Series, July, 1984.

12. W.F. Chen, Limit Analys is and Soil Plasticity, Elsevier Scientific Publishing Co., Amsterdam, Holland, 1975.

13. W.F. Chen, Plasticity in Reinforced Concrete, McGraw Hill Book Company, New York, 1982.

14. C.S. Desai and J.F. Abel, Introduction to the Finite Element Method, van Nostrand-Reinhold, New York, 1972. 
15. Y. Yamada, N. Yoshimura and T. Sakurai, "Plastic Stress-Strain Matrix and Its Application for the Solution of Elastic-Plastic Problem by the Finite Element Method", Int. J. Mech. Sci., 10, pp. 343-354, 1968.

16. D.C. Drucker and W. Prager, "Soil Mechanics and Plastic Analysis or Limit Design", Q. Appl. Math., 10, pp. 157-165, 1952.

17. A.C.T. Chen and W.F. Chen, "Constitutive Relations for Concrete', J. Eng. Mech. Div. A.S.C.E., Vol. 101, No. EM4, August, 1975.

18. B.L. Gates and P.S. Wang, "A Lisp-Based RATFOR Code Generator", Proceedings of the 3rd MACSYMA User's Conference, (ed. by E. Golden) held at General Electric Research Laboratory, Schnectady, New York, pp. 319-329, August, 1984.

19. M.C. Wirth, "On the Automation of Computational Physis", Ph.D thesis, University of California, Davis School of Applied Science, Lawrence Livermore Laboratory, Berkeley, California, 1980.

20. D.H. Lanam, "A Package for Generating and Executing Fortran Programs with MACSYMA", Master's thesis, University of California, Berkeley, CA, 1982.

21. A.C. Hern (ed), "REDUCE User's Manual, Version 3.0", the Rand Corporation, Santa Monica, CA, 1983. 


$$
\begin{aligned}
& c=\frac{E}{(1+v)(1-2 v)} \\
& \frac{1}{w}=\frac{1}{2 C}\left[(1-2 v)+6 \alpha^{2}(1+v)\right] \\
& J_{2}=\frac{1}{2} S_{i j} S_{i j}
\end{aligned}
$$

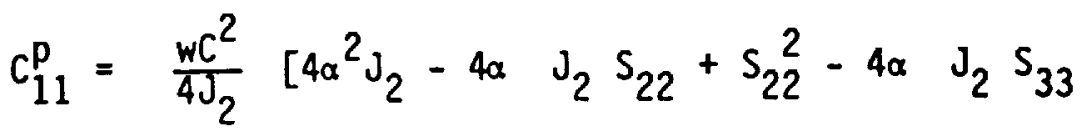

$$
\begin{aligned}
& +2 S_{22} S_{33}+S_{33}^{2}+8 \alpha^{2} v J_{2}+4 a v J_{2} S_{22}-4 v S_{22}^{2} \\
& +4 \alpha v \mathrm{~J}_{2} \mathrm{~S}_{33}-8 v \mathrm{~S}_{22} \mathrm{~S}_{33}-4 v \mathrm{~S}_{33}^{2}+4 \alpha^{2} v^{2} \mathrm{~J}_{2} \\
& +8 \alpha v^{2} \mathrm{~J}_{2} \mathrm{~S}_{22}+4 v^{2} \mathrm{~S}_{22}^{2}+8 \alpha v^{2} \cdot \mathrm{J}_{2} \mathrm{~S}_{33} \\
& \left.+8 v^{2} s_{22} s_{33}+4 v^{2} s_{33}^{2}\right] \\
& c_{12}^{p}=\frac{w c^{2}}{4 J_{2}}\left[4 \alpha^{2} J_{2}-S_{22}^{2}-2 \alpha \quad J_{2} S_{33}-S_{22} S_{33}\right. \\
& +8 \alpha{ }^{2} v \mathrm{~J}_{2}+4 v \mathrm{~S}_{22}^{2}+2 \alpha v \mathrm{~J}_{2} \mathrm{~S}_{33}+4 v \mathrm{~S}_{22} \mathrm{~S}_{33} \\
& \left.+4 \alpha^{2} v^{2} J_{2}-4 v^{2} S_{22}^{2}+4 \alpha v^{2} J_{2} S_{33}-4 v^{2} S_{22} S_{33}\right]
\end{aligned}
$$




$$
\begin{aligned}
C_{13}^{p}= & \frac{w c^{2}}{4 J_{2}}\left[4 \alpha^{2} J_{2}-s_{33}^{2}-2 \alpha J_{2} S_{22}-S_{22} S_{33}\right. \\
& +8 \alpha^{2} v J_{2}+4 v s_{33}^{2}+2 \alpha v J_{2} s_{22}+4 v S_{22} S_{33} \\
& +4 \alpha^{2} v^{2} J_{2}-4 v^{2} s_{33}^{2}+4 a v^{2} J_{2} S_{22}-4 v^{2} s_{22} S_{33}
\end{aligned}
$$

etc.

Figure 1 Typical Entries of Plastic Matrix of Drucker-Praeger Model Without the Use of Intermediate Variables. 


$$
\begin{aligned}
& a_{1}=a+\frac{1}{2 J_{2}} S_{11} \\
& a_{2}=\alpha+\frac{1}{2 J_{2}} S_{22} \\
& a_{3}=\alpha+\frac{1}{2 J_{2}} S_{33} \\
& a_{4}=\frac{1}{J_{2}} S_{12} \\
& a_{5}=\frac{1}{J_{2}} S_{23} \\
& a_{6}=\frac{1}{J_{2}} S_{31} \\
& f_{i}=(1-2 v) a_{i}+v a_{0}, \quad i=1,2, \text { or } 3 \\
& a_{0}=a_{1}+a_{2}+a_{3} \\
& f_{j}=\frac{(1-2 v)}{2} \cdot a_{j}, j=4,5, \text { or } 6
\end{aligned}
$$

Figure 2 Intermediate Variables for Expression Simplification of Drucker-Praeger Model 


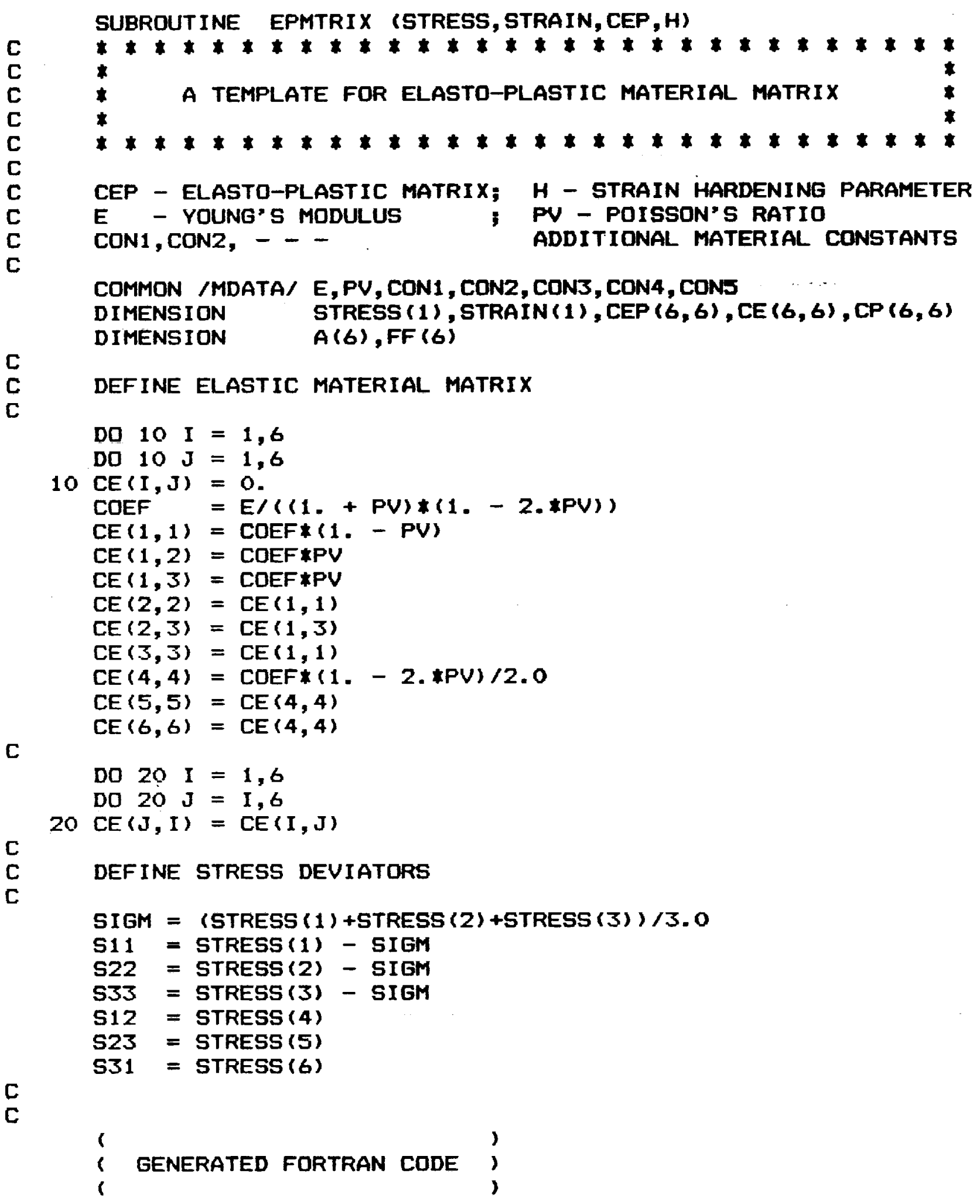

RETURN

END

Figure 3 A Template File for Elasto-Plastic Material Matrix 


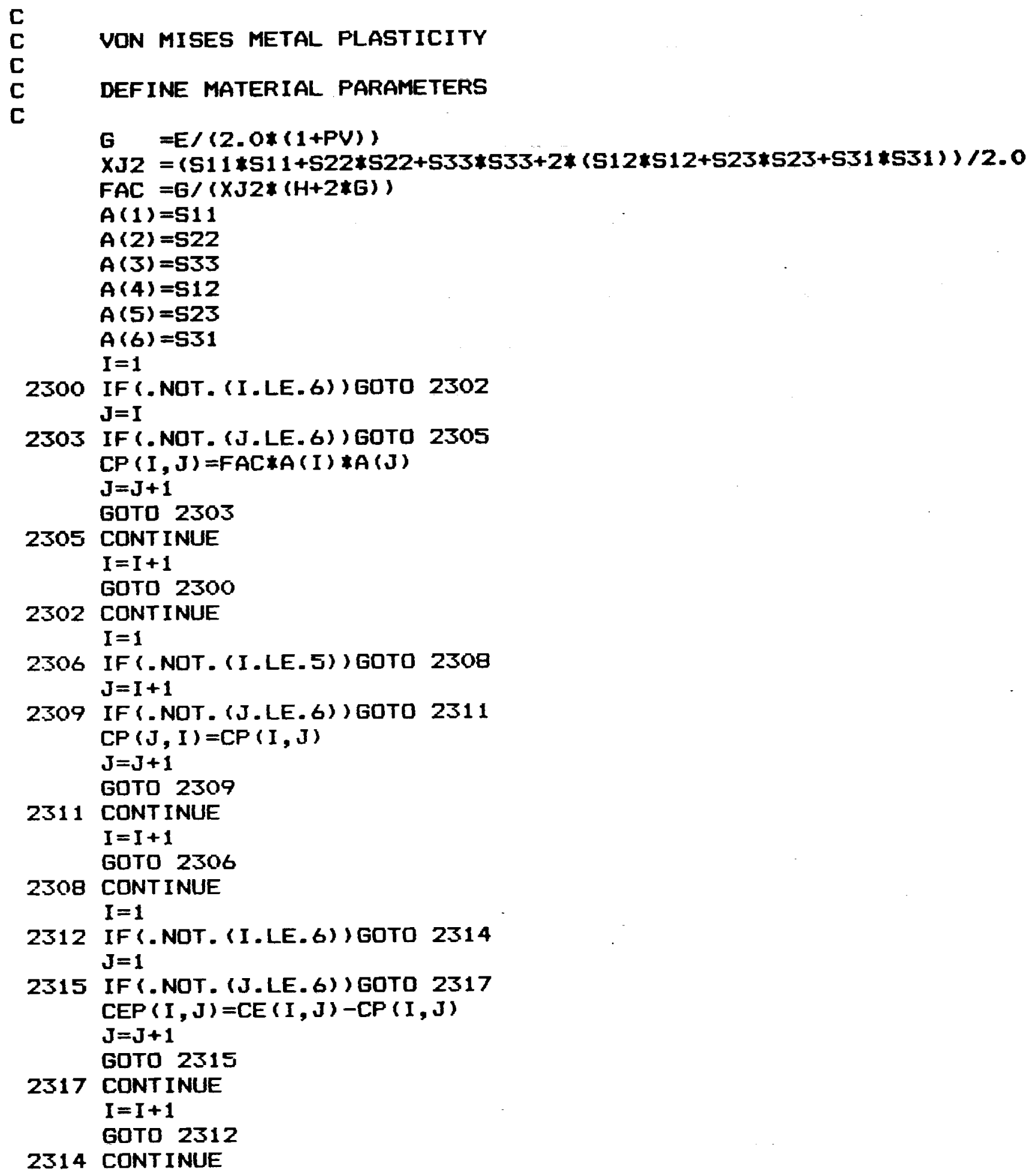




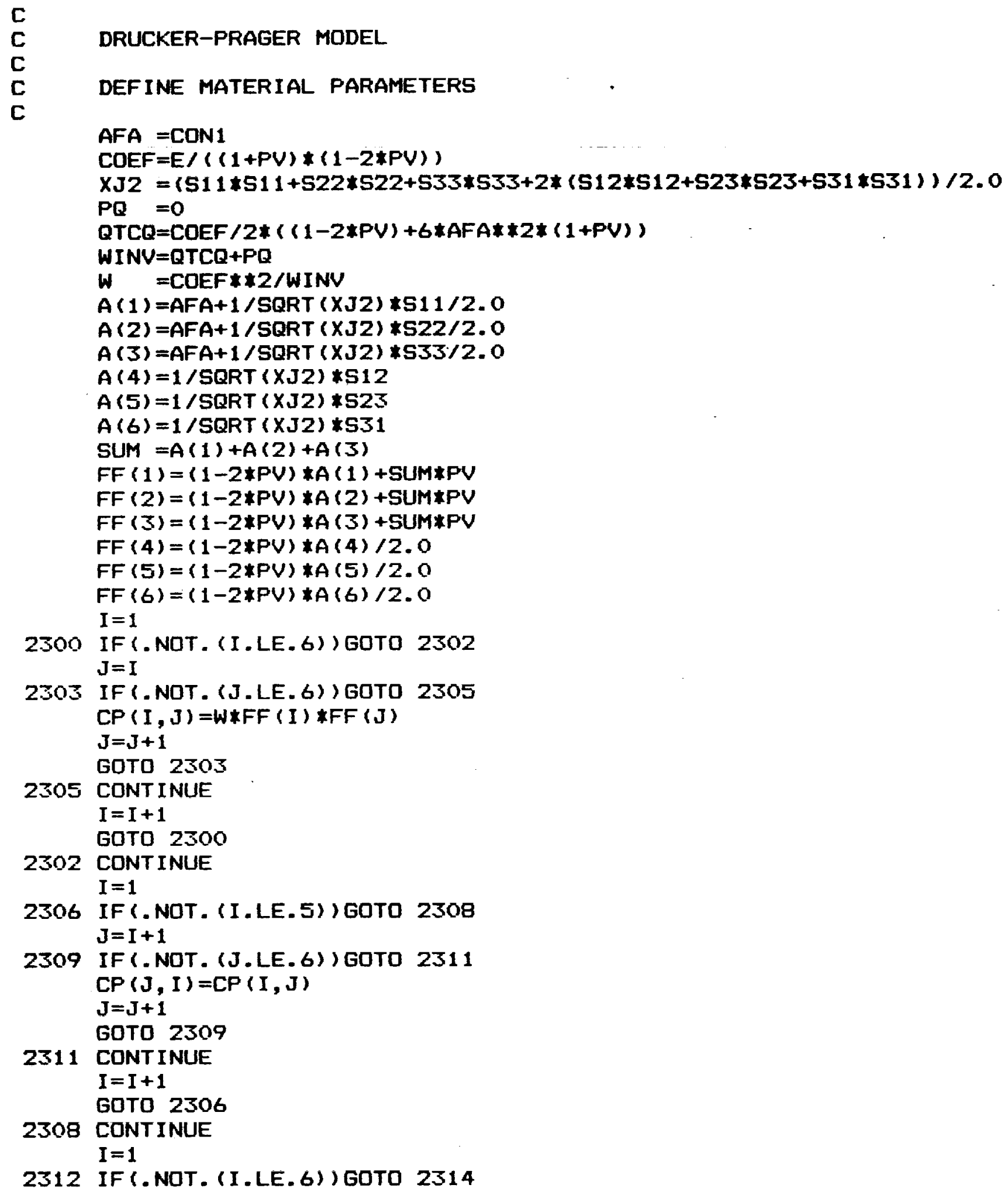




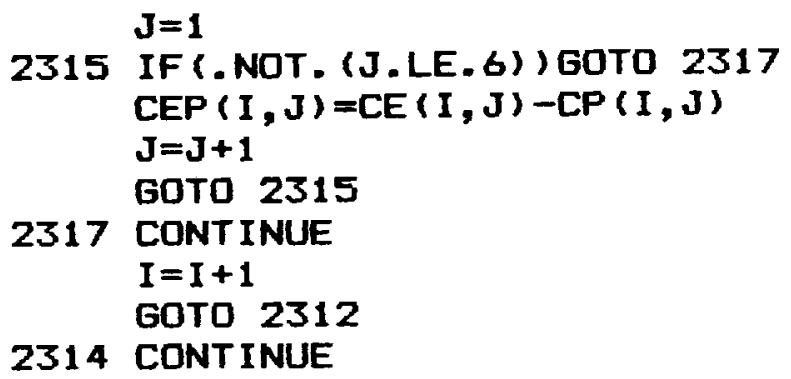

A-3 


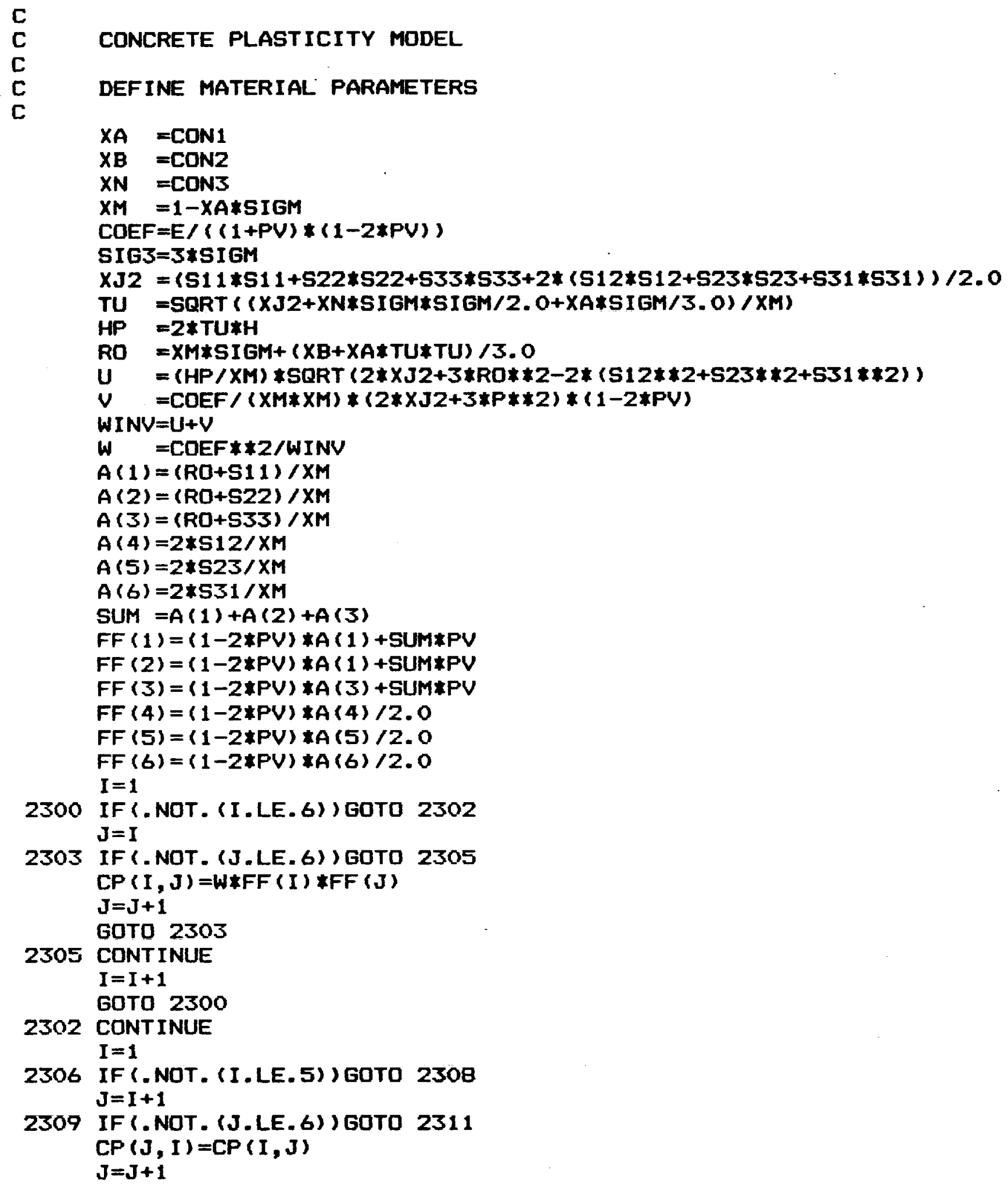




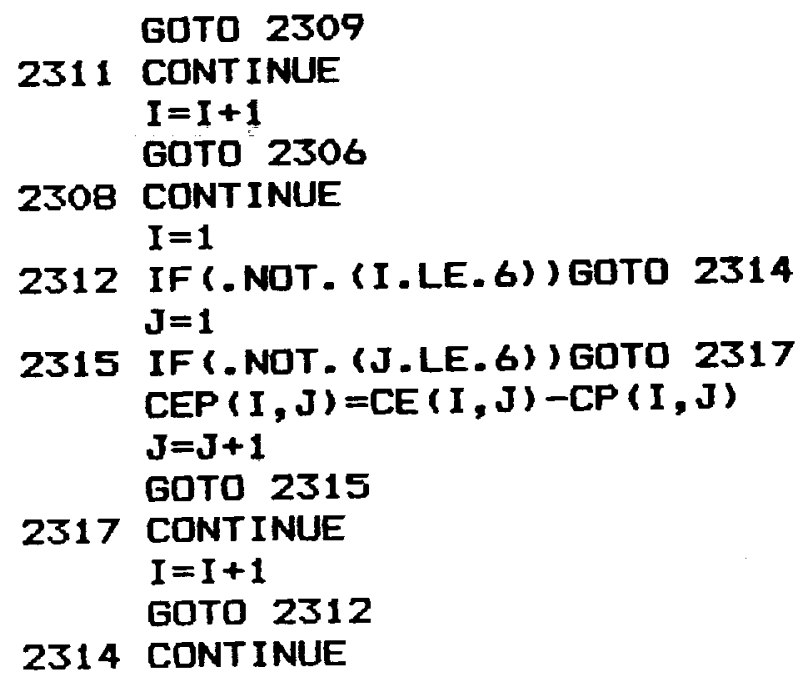

2314 CONTINUE 


\begin{tabular}{|c|c|c|}
\hline \multicolumn{2}{|c|}{ REPORT DOCUMENTATION PAGE } & $\begin{array}{l}\text { Form Approved } \\
\text { OMB No. 0704-0188 }\end{array}$ \\
\hline \multicolumn{3}{|c|}{ 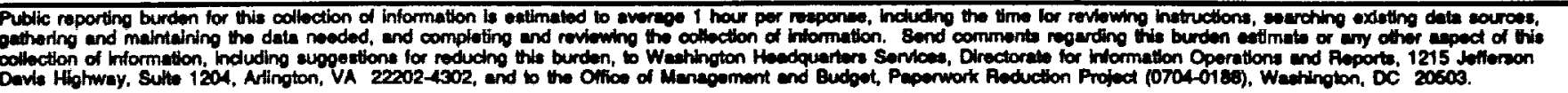 } \\
\hline 1. AGENCY USE ONLY (LoAve blank) & $\begin{array}{l}\text { 2. REPOAT DATE } \\
\text { October } 1991\end{array}$ & $\begin{array}{l}\text { ND DATES COVERED } \\
\text { Final Contractor Report }\end{array}$ \\
\hline \multicolumn{2}{|c|}{$\begin{array}{l}\text { 4. ThE AND SUBTITLE } \\
\text { On the Symbolic Manipulation and Code Generation for Elasto-Plastic } \\
\text { Material Matrices }\end{array}$} & 5. FUNDING NUMBERS \\
\hline \multicolumn{2}{|c|}{$\begin{array}{l}\text { AUTHOR(S) } \\
\text { T.Y. Chang, A.F. Saleeb, P.S. Wang, and H.Q. Tan }\end{array}$} & T.Y. Chang, A.F. Saleeb, P.S. Wang, and H.Q. Tan \\
\hline \multicolumn{2}{|c|}{$\begin{array}{l}\text { University of Akron } \\
\text { Department of Civil Engineering } \\
\text { Akron, Ohio } 44325\end{array}$} & $\begin{array}{l}\text { - PERFORMING ORGANIZATION } \\
\text { REPORT MUMBER } \\
\text { NONE }\end{array}$ \\
\hline \multicolumn{2}{|c|}{$\begin{array}{l}\text { National Aeronautics and Space Administration } \\
\text { Lewis Research Center } \\
\text { Cleveland, Ohio } 44135-3191\end{array}$} & $\begin{array}{l}\text { 10. SPONSORING/MONTOAING } \\
\text { AGENCY REPOAT NUMBER } \\
\text { NASA CR-187223 }\end{array}$ \\
\hline \multicolumn{3}{|c|}{$\begin{array}{l}\text { Project Manager, C.C. Chamis, Structures Division, NASA Lewis Research Center, (216) } 433-3252 \text {. T.Y. Chang } \\
\text { and A.F. Saleeb, University of Akron; P.S. Wang and H.Q. Tan, Kent State University, Department of Mathematics, } \\
\text { Kent, Ohio } 44242 \text {. }\end{array}$} \\
\hline $\begin{array}{l}\text { 12.. DISTAIBUTION/AVAILABILITY S } \\
\text { Unclassified - Unlimited } \\
\text { Subject Category } 39\end{array}$ & EMENT & 12b. DHSTRIBUTION CODE \\
\hline
\end{tabular}

13. ABSTRACT (Mox/mum 200 words)

A computerized procedure for symbolic manipulations and FORTRAN code generation of elasto-plastic material matrix for finite element applications is presented. Special emphasis is placed on expression simplifications during intermediate derivations, optimal code generation and interface with the main program. A systematic procedure is outlined to avoid redundant algebraic manipulations. Symbolic expressions of the derived material stiffness matrix are automatically converted to RATFOR code which is then translated into FORTRAN statements through a preprocessor. To minimize the interface problem with the main program, a template file is prepared so that the translated FORTRAN statements can be merged into the file to form a subroutine (or a submodule). Three constitutive models; namely, von Mises plasticity, Drucker-Prager model, and a concrete plasticity model, are used as illustrative examples.

\begin{tabular}{|c|c|c|c|}
\hline \multicolumn{3}{|c|}{ 14. SUBJECT TEAMS } & \begin{tabular}{|c|} 
15. NUMBEA OF PAGES \\
28 \\
16. PAICE CODE \\
A03 \\
\end{tabular} \\
\hline $\begin{array}{l}\text { 17. SECUATYY CLASSIFICATION } \\
\text { OF REPORT } \\
\text { Unclassified }\end{array}$ & $\begin{array}{l}\text { 18. SECURITY CLASSIFICATION } \\
\text { OF THIS PAGE } \\
\text { Unclassified }\end{array}$ & $\begin{array}{l}\text { 19. SECURTY CLASSIFICATION } \\
\text { OF ABSTHACT } \\
\text { Unclassified }\end{array}$ & 20. LIMITATION OF ABSTRACT \\
\hline
\end{tabular}

